\title{
Impedance Measurements With Second-Order Harmonic Oscillator for Testing Food Sterility
}

\author{
Stoyan N. Nihtianov, Senior Member, IEEE, Georgi P. Shterev, Nikolay Petrov, and \\ Gerard C. M. Meijer, Senior Member, IEEE
}

\begin{abstract}
In this paper, we present a simple interface circuit for measuring impedance based on a second-order harmonic oscillator. The circuit is intended for testing the sterility of aseptically packed food products, where it must measure the conductivity changes of the packaged food in a nondestructive way. The measured impedance is modeled as a low-ohmic resistor (representing the conductivity of the food) in series with a capacitor (the walls of the food container). The oscillator is built with fast current-feedback operational amplifiers (CFOA). To measure the resistive component of the impedance, an AC-to-DC converter is realized with a wide frequency range analog multiplier. The presented experimental results prove that with this interface circuit an accurate measurement of the impedance components can be achieved in a frequency range up to $10 \mathrm{MHz}$. The range of the resistive component is from a few ohms up to $200 \mathrm{ohms}$; the range of the capacitive component is from $50 \mathrm{pF}$ up to $300 \mathrm{pF}$.
\end{abstract}

\section{INTRODUCTION}

C URRENTLY, the most popular way to measure impedance is to apply the auto-balanced bridge technique, also called the vector-voltage-current ratio measurement method [1], [2]. This principle is used in impedance analyzers and RLC meters because of its good accuracy over a wide frequency range [3]. The principle requires at least three sophisticated functional blocks: a tunable harmonic oscillator, an auto-balanced bridge, and a synchronous phase detector. For sensor-interfacing purposes, this solution appears to be quite expensive and complicated. Attempts have been made to optimize and simplify the above-mentioned technique [4] and alternative methods have been sought to interface impedance sensors [5]-[8], but all of them are applicable in a frequency range below $100 \mathrm{kHz}$.

This paper introduces a novel application-specific impedance-measurement technique for the frequency range up to $10 \mathrm{MHz}$. This technique uses a second-order harmonic oscillator and is developed for the needs of nondestructive sterility testing of aseptically-packaged, long shelf-life food products [9], [10]. The technique is based on measurement of the conductivity changes that accompany the growth of a large number of bacterial species in food. Because our goal is testing a big part of the production lot and early detection of spoiled

Manuscript received May 4, 2000; revised February 22, 2001. This work was supported by the Dutch Foundation for Technical Sciences (STW), Project DEL.66.4369.

S. N. Nihtianov and G. C. M. Meijer are with the Faculty of Information Technology and Systems, Delft University of Technology, Delft, The Netherlands (e-mail: S.Nihtianov@ITS.TUDelft.NL).

G. P. Shterev and N. Petrov are with the Electronics and Automatics Department, Technical University, Sofia, Branch Plovdiv, Plovdiv, Bulgaria.

Publisher Item Identifier S 0018-9456(01)07352-1.
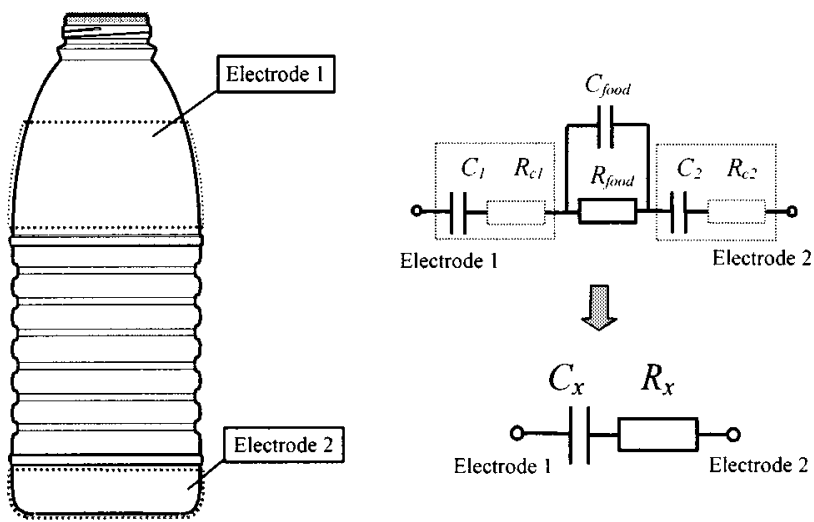

Fig. 1. Plastic bottle with two external electrodes for nondestructive sterility testing and the equivalent electric circuit of the measured impedance.

food, we are interested in the accurate and fast measurement of the resistive component of the unknown impedance, which is in series with a capacitive component due to the walls of the package. The value of the capacitive component is also informative and is used to test the quality of the food container. The best frequency to sense the conductivity changes of the food vary with the type of the food container, the electrode setting, and the conductivity of the sterile food.

Fig. 1 shows a typical food container for long shelf-life food-a plastic bottle, blow-molded from high-density polyethylene, and the equivalent electric circuit of the measured impedance. Two external electrodes are used to sense the conductivity changes of the food. The values of the coupling capacitors $C_{1}$ and $C_{2}$, and their loss resistances $R_{c 1}$ and $R_{c 2}$, depend on the electrical properties and the thickness of the polyethylene and on the surface of the electrodes. $R_{\text {food }}$ and $C_{\text {food }}$ represent the electrical properties of the food.

The best sensitivity for $R_{\text {food }}$ can be achieved at the frequency at which the influence of the loss resistance in the coupling capacitors $\left(R_{c 1}\right.$ and $\left.R_{c 2}\right)$ and the dielectric properties of the food $\left(C_{\text {foodl }}\right)$ are the smallest and we can neglect them. At these frequencies, the equivalent electric circuit (see Fig. 1) can be simplified to one resistor $R_{x} \approx R_{\text {food }}$ and one capacitor $C_{x} \approx C_{1} C_{2} /\left(C_{1}+C_{2}\right)$, in series. For example, with the setting in Fig. 1, the coupling capacitors $C_{1}$ and $C_{2}$ have values of approximately $250 \mathrm{pF}, R_{\text {food }}$ for sterile UHT-milk is about $50 \Omega$, and the best testing frequency is between $1 \mathrm{MHz}$ and $2 \mathrm{MHz}$. The $R_{\text {food }}$ of fish sauce is about $20 \Omega$, and the best testing frequency is between $3 \mathrm{MHz}$ and $5 \mathrm{MHz}$.

Section II discusses the principle of operation of the interface circuit. In Section III, the measurement setup is presented. In 


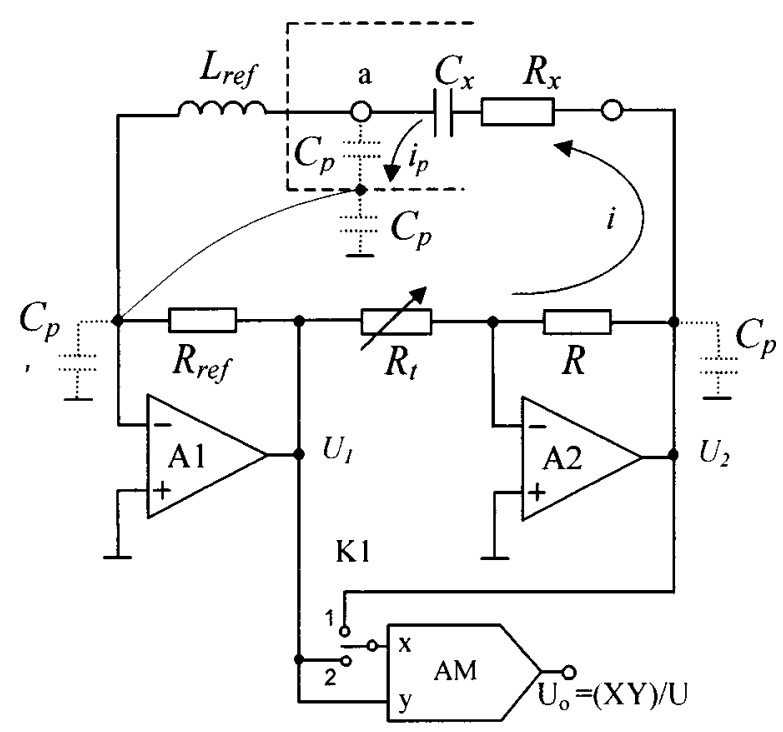

Fig. 2. Simplified impedance interface circuit.

Section IV, the experimental results are presented and discussed. Finally, the conclusions are given in Section V.

\section{PRINCIPLE OF OPERATION}

To build a second-order oscillator, we need two basic blocks - a frequency-selective network (resonator) and an undamping circuit that compensates for the energy loss in the resonator and ensures continuous oscillations. Fig. 2 shows a simplified circuit, diagram of the impedance sensor interface. A natural choice for the resonator is a serial RLC circuit in which $R_{x}$ and $C_{x}$ are the components of the unknown impedance and $L_{\text {ref }}$ is a reference inductor. As an undamping circuit, two inverting stages are used. In this way, low-ohmic input and output are ensured, which eliminates the influence of the parasitic capacitors $C_{p 1}$ and $C_{p 2}$ at both ends of the resonator. Assuming that the amplifiers A1 and A2 from Fig. 2 are ideal (infinite gain and unlimited bandwidth), the circuit will start oscillating at a frequency that is equal to the resonance frequency of the resonator

$$
\omega_{\circ}=1 / \sqrt{C_{x} L_{\mathrm{ref}}}
$$

if

$$
\left(R_{\mathrm{ref}} R_{1}\right) /\left(R_{x} R_{t}\right) \geq 1
$$

To ensure stable sine oscillations for an arbitrary value of $R_{x}$, we have to tune one of the other three resistors automatically (in Fig. 2 this is $R_{t}$ ) in such a way that it is always true that

$$
\left(R_{\mathrm{ref}} R_{1}\right) /\left(R_{x} R_{t}\right)=1
$$

The existence of a parasitic capacitance $C_{p}$ (not shown in Fig. 2) between point a, at which $L_{\text {ref }}$ and the measured impedance are connected, and ground will cause a part $\left(i_{p}\right)$ of the loop current $i$ to be directed to ground. This will result in a change of the oscillating frequency

$$
\omega_{o}=1 / \sqrt{L_{\mathrm{ref}}\left(C_{x}+C_{p}\right)}
$$

and will result in a relative error for $R_{x}$ of

$$
\varepsilon_{R_{x}}=-\frac{C_{p}}{C_{p}+C_{x}}
$$

If the value of this parasitic capacitance is too high, one can use an electric-field shield that is connected to the inverting input of A1 (see Fig. 2); in this way, the initial parasitic capacitance $C_{p}$ is split in two parts $-C_{p 3}$ and $C_{p 4}$. $C_{p 4}$ will appear in parallel to $C_{p 1}$ and its influence will be reduced significantly, just like that of $C_{p 1}$. The leakage current $\boldsymbol{i}_{p}$ through $C_{p 3}$ will be added to the loop current $i$ and the error for $R_{x}$ (5) will be eliminated. What remains is an offset error for $C_{x}$, which can be eliminated by applying, for example, a multi-signal measurement technique.

The value of $R_{x}$ can be obtained in several ways. When resistors $R_{\text {ref }}$ and $R_{1}$ are known, the only value we need to calculate $R_{x}$ is the value of $R_{t}$. At lower frequencies, we could use a digital potentiometer as $R_{t}$. In this way, the circuit will automatically generate a digital code at the control input of the potentiometer, which can be used as a measure for $R_{x}$. Unfortunately, the big parasitic capacitance at the signal inputs of existing digital potentiometers would pose a serious problem at higher frequencies. Another alternative is to use a MOSFET as a controlled resistor. The dc voltage at the gate of the MOSFET can also be used as a measure for $R_{x}$, but then we have to deal with nonlinearity problems caused by the nonlinear behavior of the MOSFET as a voltage-controlled resistor and the temperature dependence of its parameters.

Our choice is to use the MOSFET-transistor as a voltagecontrolled resistor, but to measure the value of $R_{x}$ with a highfrequency analog multiplier (AM in Fig. 2). The measurement is carried out in two steps. First, with switch $\mathrm{K} 1$ in position 1 (see Fig. 2), we measure the dc component of the multiple of $U_{1}$ and $U_{2}$

$$
U_{o 1}=\frac{U_{1} U_{2}}{U}
$$

and then, with switch $\mathrm{K} 1$ in position 2, the dc component of the square value of $U_{1}$

$$
U_{o 2}=\frac{U_{1}^{2}}{U} .
$$

In (6) and (7), the $U_{o 1(2)}$ are the dc components of the output voltage of the multiplier and $U$ is a reference dc voltage. The ratio between (6) and (7) equals the ratio between $U_{2}$ and $U_{1}$, which in turn is equal to $R_{x} / R_{\text {ref }}$

$$
\frac{U_{o 1}}{U_{o 2}}=\frac{U_{2}}{U_{1}}=\frac{R_{x}}{R_{\text {ref }}} .
$$

From (8), the value of $R_{x}$ can be determined by

$$
R_{x}=R_{\text {ref }} \frac{U_{o 1}}{U_{o 2}}
$$

There are three basic advantages to measure $R_{x}$, as shown above. First of all, by using the ratio of the two output voltages of the analog multiplier we eliminate its multiplicative error, which could be significant at high frequencies. Secondly, the effect of the harmonic distortion caused mainly by the voltage- 


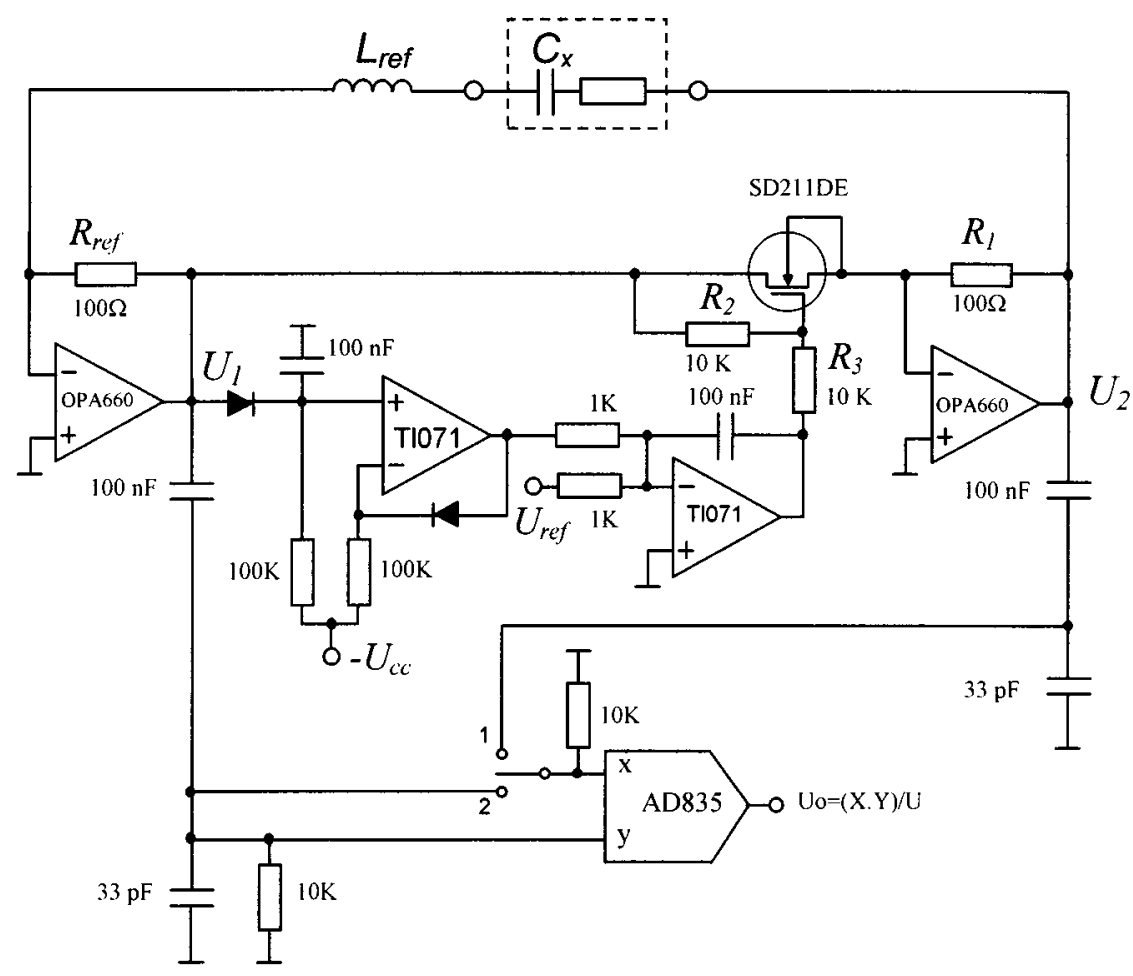

Fig. 3. Circuit diagram of the impedance sensor interface with which the experimental results were obtained.

controlled resistor $R_{t}$ is considerably reduced. The harmonic distortion of the signal at the output of A2 $\left(U_{2}\right)$ is highest at the highest value of $R_{t}$. At the same time, the resonator acts as a filter for the high-level harmonics and its best performance is when $R_{x}$ has its smallest value and $R_{t}$ has its largest value. When $U_{1}$ is multiplied with $U_{2}$, only the first harmonic of $U_{2}$ has an impact on the output dc signal.

A third advantage is related to a certain compensation of the error due to the phase shift $\varphi_{\mathrm{A}}=\varphi_{\mathrm{A} 1}+\varphi_{\mathrm{A} 2}$ introduced by the amplifiers A1 and A2. This phase shift causes the oscillator to oscillate at frequency $\omega_{g}$, which differs from the resonance frequency of the resonator $\omega_{0}$ with

$$
\omega_{g} \approx \omega_{o}\left(1+\frac{\varphi_{A}}{2 Q}\right)
$$

where $Q$ is the quality factor of the resonator. At the same time, when a phase shift of $\varphi_{\mathrm{A} 2}$ between $U_{1}$ and $U_{2}$ occurs, (6) becomes

$$
U_{o 1}=\frac{U_{1} U_{2} \cos \left(\varphi_{\mathrm{A} 2}\right)}{U}
$$

Taking into account the phase shift introduced by the amplifiers, we can show that (9) changes into

$$
R_{x}=R_{\text {ref }} \frac{U_{o 1}}{U_{o}} \cos \left(\varphi_{\mathrm{A} 2}\right) \sqrt{1+\tan ^{2}\left(\varphi_{\mathrm{A} 1}+\varphi_{\mathrm{A} 2}\right)} .
$$

From (12), it is easily seen that the full compensation effect is achieved when $\varphi_{\mathrm{A} 1} \ll \varphi_{\mathrm{A} 2}$.

The value of $R_{x}$ directly affects the quality factor $Q$. To ensure that the oscillating frequency $\omega_{g}$, from which we calculate
$C_{x}$, remains independent of the value of $R_{x}$, we have to use amplifiers with a very small phase shift in the measurement frequency range, as shown in (10).

\section{MEASUREMENT SETUP}

Experiments were carried out with the circuit shown in Fig. 3. For a reference inductance $L_{\text {ref }}$, we used molded inductors IM-6-38 (Dale Electronics, Inc.) with the features: small package, epoxy molded construction with superior moisture protection, high $Q$ and self-resonant frequency, low DC resistance, and an insulation resistance higher than $1000 \mathrm{M} \Omega$.

The undumping circuit is realized with two wide frequency bandwidth $\left(f_{-3 \mathrm{~dB}} \approx 850 \mathrm{MHz}\right)$ current-feedback operational amplifiers OPA 660 (Burr Brown). A current-feedback operational amplifier (CFOA) is the best choice in our application for two reasons. First, it has a high-frequency performance that is superior to the performance of the voltage-feedback amplifiers (a higher cut-off frequency, $f_{-3 \mathrm{~dB}}$ ). This means we have a lower phase shift $\varphi_{A}$ in our measurement frequency range. Secondly, the cut-off frequency and the phase shift $\varphi_{A}$ change only slightly for different values of $R_{x}$. Although a multiplicative error is introduced, the transfer characteristic stays linear and the phase-shift error in (11) can be cancelled by using the multi-signal measurement technique. The relatively high input current noise of a CFOA (a few tens of $\mathrm{pA} / \sqrt{ } \mathrm{Hz}$ ) is not a serious problem in this application because the loop current we use is in the milliampere range and, besides, the output noise of A2 is filtered by the resonance circuit.

The automatic gain-control circuit (AGC) is realized with two OpAmp TL071 (TI) stages, and the MOSFET SD211DE (Siliconix) is used as a voltage-controlled resistor. By keeping the amplitude of $U_{1}$ constant, the AGC circuit fixes the loop current 
TABLE I

Measured Relative Power of the SeCOND AND the THRID HARMONIC OF $U_{1}$ AND $U_{2}$

\begin{tabular}{c|c|c|c|c|c}
\hline \multicolumn{2}{c|}{} & \multicolumn{2}{|c|}{ Harmonics of $U_{l}$} & \multicolumn{2}{c}{ Harmonics of $U_{2}$} \\
\hline \multirow{2}{*}{$2, \mathrm{MHz}$} & $\mathrm{Rx}, \Omega$ & $2^{\text {nd }}, \mathrm{dBm}$ & $3^{\text {rd }}, \mathrm{dBm}$ & $2^{\text {nd }}, \mathrm{dBm}$ & $3^{\text {rd }}, \mathrm{dBm}$ \\
\hline \multirow{2}{*}{2} & 50 & -42 & -39 & -26 & -30 \\
\cline { 2 - 6 } & 100 & -44 & -45 & -30 & -40 \\
\hline \multirow{2}{*}{6} & 50 & -32 & -43 & -19 & -30 \\
\cline { 2 - 6 } & 100 & -35 & -49 & -30 & -46 \\
\hline \multirow{2}{*}{9} & 50 & -30 & -42 & -20 & -38 \\
\cline { 2 - 6 } & 100 & -31 & -47 & -27 & -45 \\
\hline
\end{tabular}

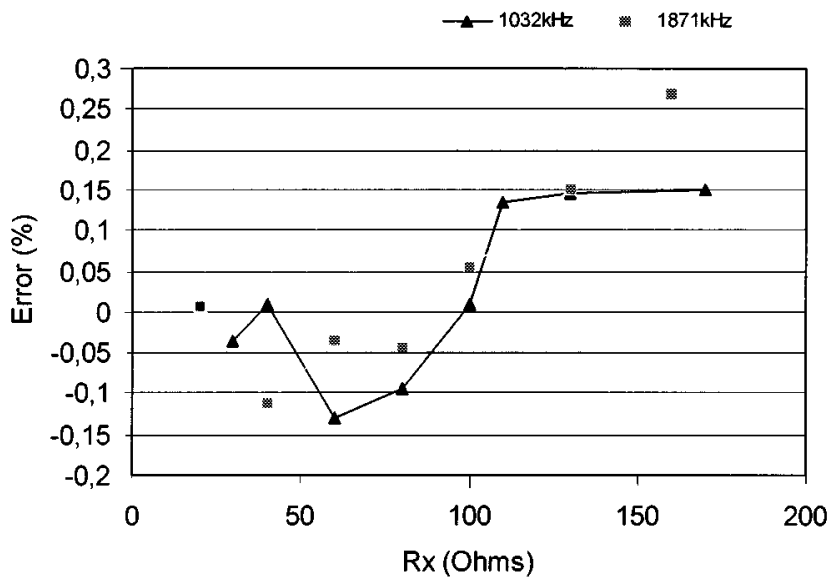

Fig. 4. Relative error of the measured resistive component for lower frequencies.

at $i \approx U_{1} / R_{\text {ref }}$ so it is independent of the value of $R_{x}$. With resistor $R_{2}$ and $R_{3}$, the linear operating region of the MOSFET is extended [11].

For the ac-to-dc conversion, we use the four-quadrant, voltage-output multiplier AD835 (Analog Devices). It has an internal reference voltage $U$ with a nominal value of 1.05 $\mathrm{V}$ and $-3 \mathrm{~dB}$ output bandwidth of $250 \mathrm{MHz}$. The inputs of the analog multiplier are capacitively coupled to the outputs of the amplifiers A1 and A2. For the biasing of AD835, two resistors of $10 \mathrm{k} \Omega$ are used in parallel with capacitors of $33 \mathrm{pF}$, which produces an output offset voltage of about $52 \mathrm{mV}$. The capacitors are used to prevent the circuit from oscillating at frequencies higher than $10 \mathrm{MHz}$.

\section{EXPERIMENTAL RESULTS}

With a spectrum analyzer, the harmonic distortion levels of $U_{1}$ and $U_{2}$ (see Fig. 3) were measured for two values of $R_{x}: 50 \Omega$ and $100 \Omega$, and for an amplitude of $U_{1}$ of $0.75 \mathrm{~V}$. Table I presents the results for the measured relative power of the second and the third harmonic of $U_{1}$ and $U_{2}$ with respect to the power of the first harmonic. As one can see, the distortion level of $U_{2}$ is quite low, and it is further reduced by the filtering effect of the resonator. Fig. 4 shows the relative error of the measured resistive component $R_{x}$ of the impedance versus its value for two frequencies: $1032 \mathrm{kHz}$ and $1871 \mathrm{kHz}$. The measurement range of the resistive component was from $30 \Omega$ to $170 \Omega$.

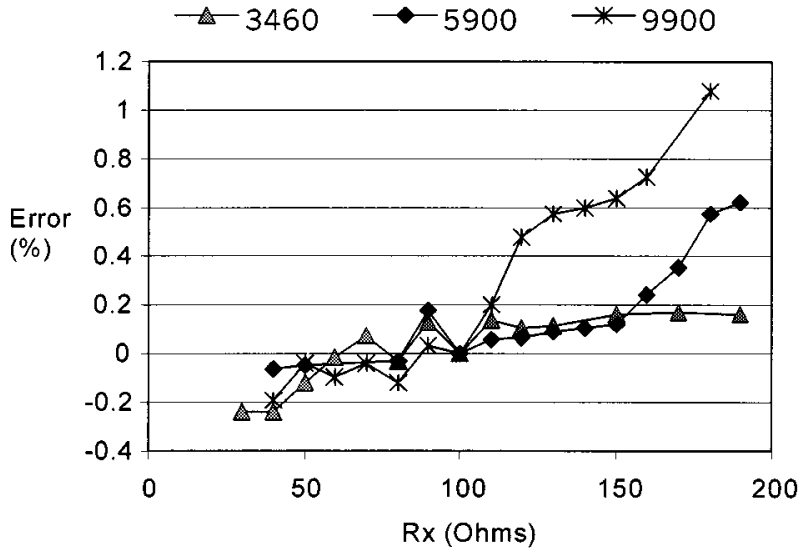

Fig. 5. Relative error of the measured resistive component $R_{x}$ at higher frequencies.

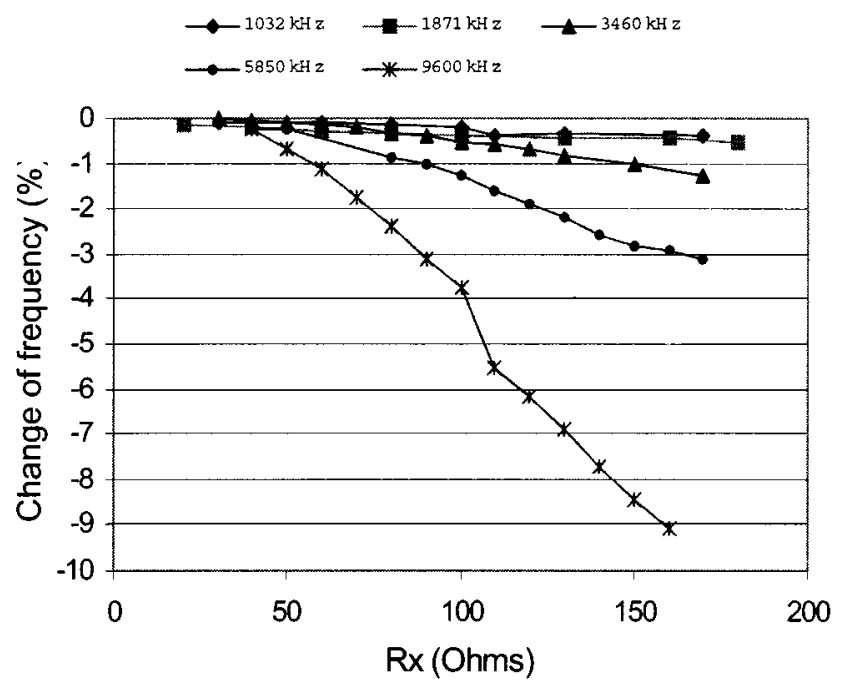

Fig. 6. Dependence of the oscillating frequency on the value of $R_{x}$.

As a reference, impedance analyzer (HP4194A) was used, with an error specification for this frequency range that does not exceed $0.125 \%$. The measurement results showed a maximum error of $\pm 0.15 \%$ for a frequency of $1032 \mathrm{kHz}$ and a maximum error of $+0.3 \%$ and $-0.2 \%$ for a frequency of $1871 \mathrm{kHz}$.

For higher frequencies, the impedance analyzer was not suitable as a reference measurement instrument since its error specification at $10 \mathrm{MHz}$ is already above $1 \%$. For this reason, we took as reference our result for $R_{x}=100 \Omega$ and by changing the DC value of $R_{x}$ by $10 \Omega$, we covered the range from $30 \Omega$ to $200 \Omega$. Fig. 5 shows the relative error that we measured with the relative changes of $R_{x}$. For values of $R_{x}$ below $100 \Omega$, which is the range that is most interesting for us, the error is within $\pm 0.2 \%$. Above $100 \Omega$, the error starts to grow. The highest value is $1.1 \%$ for a frequency of $9.9 \mathrm{MHz}$ and $R_{x}=180 \Omega$. The errors displayed in Figs. 4 and 5 have a clear systematic component, which can be explained with (11). For higher values of $R_{x}$ and lower values of $R_{t}$, the phase shift $\varphi_{\mathrm{A} 1}$ of the stage A1 is slightly growing, and the phase shift $\varphi_{\mathrm{A} 2}$ of the stage A 2 is decreasing, which ensures better frequency compensation. The systematic error, especially for values of $R_{x}$ above $100 \Omega$, can be further reduced by using, for example, an appropriate read-out table. 
Fig. 6 shows the dependence of the oscillating frequency on the value of the resistive component $R_{x}$. This dependence means that the measured value of $C_{x}$ is a function of the value of $R_{x}$. For frequencies up to $2 \mathrm{MHz}$, the frequency changes only $\pm 0.25 \%$ for $R_{x}$ from $30 \Omega$ to $170 \Omega$. With an increase in frequency, the same small variation of the frequency is achieved for a reduced range of $R_{x}$. In other words, if the value of $R_{x}$ is higher, the measurement frequency must be lower in order to keep the error in determining $C_{x}$ small. This is, of course, a natural choice for many applications, including the one presented in this article. An alternative solution is to compensate the error in determining $C_{x}$ by applying a multi-signal or statistical error-correction measurement technique.

\section{CONCLUSION}

A simple, self-oscillating circuit for impedance measurement is presented. It is intended to test the sterility of aseptically packed food products by measuring the conductivity changes of the packaged food in a nondestructive way. The oscillator has been built with fast current-feedback operational amplifiers. To measure the resistive component of the impedance, an AC-to-DC converter with a wide frequency range analog multiplier is used.

The advantages of this interface circuit are: i) an external oscillator is not needed, ii) synchronous detection is not necessary, and iii) at resonance frequency, the reactive components compensate each other to ensure the highest possible sensitivity for the resistive component. The experimental results presented prove that with this interface circuit, an accurate measurement of the impedance components can be achieved in a frequency range up to $10 \mathrm{MHz}$.

\section{REFERENCES}

[1] D. Marioli, E. Sardini, and A. Taroni, "High-accuracy measurement techniques for capacitance transducers," Meas. Sci. Technol., vol. 4, pp. 337-343, 1993.

[2] W. Q. Yang, "A self-balancing circuit to measure capacitance and loss conductance for industrial applications," IEEE Trans. Instrum. Meas., vol. 45, pp. 955-958, Dec. 1996.

[3] Service Manual, Impedance/Gain-Phase Analyzer Model 4194A, 1986.

[4] C. Kolle and P. O'Leary, "Low-cost, high-precision measurement system for capacitive sensors," Meas. Sci. Technol., vol. 9, pp. 510-517, 1998.

[5] M. A. Atmanand, V. J. Kumar, and V. G. K. Murti, "A microcontrollerbased scheme for measurement of $L$ and $C$," Meas. Sci. Technol., vol. 6, pp. 576-581, 1995.

[6] G. M. Caranti and M. A. Re, "A vector impedance meter digitally controlled," Review Sci. Instrum., vol. 62, pp. 3092-3097, Dec. 1991.

[7] S. M. R. Taha, "Digital measurement of the polar and rectangular forms of impedances," IEEE Trans. Instrum. Meas., vol. 38, pp. 59-63, Feb. 1989.

[8] L. Angrisani, A. Baccigalupi, and A. Pietrosanto, "A digital signal-processing instrument for impedance measurement," IEEE Trans. Instrum. Meas., vol. 45, pp. 930-934, Dec. 1996.
[9] S. N. Nihtianov and G. C. M. Meijer, "Non-destructive on-line sterility testing of long-shelf-life aseptically packed food products by impedance measurements," Proc. IEEE Autotestcon'99, pp. 243-249, Aug. 30-Sept. 21999.

[10] — "Indirect conductivity measurement of liquids in flexible containers," in Proc. Instrum. Meas. Tech. Conf., IMTC/97, vol. 2, Ottawa, ON, Canada, May 19-21, 1997, pp. 919-922.

[11] A. Bilotti, "Operation of a MOS transistor as a variable resistor," Proc. IEEE, vol. 54, pp. 1093-1094, Aug. 1966

Stoyan N. Nihtianov (SM'00) was born in Bulgaria. He received the M.Sc. and $\mathrm{Ph}$.D. degrees in electronics from the Technical University, Sofia, Bulgaria, in 1980 and 1987, respectively.

From 1987 until 1995, he was part of the Department of Electronics, Technical University, Sofia, where he was an Assistant Professor and, later, an Associate Professor, engaged in research and teaching on analogue circuits and smart sensors. In 1995, he joined the Laboratory of Electronics, University of Technology, Delft, The Netherlands, where he was a Senior Scientist and did research on nondestructive methods and electronic equipment for on-line sterility testing of aseptically packaged foods. Since August 2000, he has been a competence leader of the Sensor Interface Electronics Team of ASML, Veldhoven, The Netherlands.

Georgi P. Shterev graduated from the Technical University, Sofia, Bulgaria, in 1978, with a specialty in electrical measurement technique.

He worked at the Bulgarian State Agency of Standardization and Metrology from 1978 to 1986. At the moment, he is part of the Technical University, Sofia, Branch Plovdiv. He is a Senior Lecturer and he is teaching: electrical measurements, measurement of non-electrical quantities, and quality control. His scientific research interests are in the field of sensors and transducers-pneumatic transducers for geometrical quantities. He is also working in the field of data processing and metrologic securing of the manufacture.

Nikolay Petrov graduated from the Technical University, Sofia, Bulgaria, Branch Plovdiv, in 2000, with a major in electronic and laser technique.

His scientific research work is in the field of analogue circuitry and power electronics.

Gerard C. M. Meijer (SM'99) was born in Wateringen, The Netherlands, on June 28, 1945. He received the ingenieurs (M.S.) and Ph.D. degrees in electrical engineering from the Delft University of Technology, Delft, The Netherlands, in 1972 and 1982, respectively.

Since 1972, he has been part of the faculty of Electrical Engineering of Delft University of Technology, where he is an Associate Professor engaged in research and teaching on smart sensor systems and analog electronics. In 1984, and part-time during 1985-1987, he was seconded to the Delft Instruments Company where he was involved in the development of industrial level gauges and temperature transducers. In 1996, he was one of the founders of the company SensArt, where he is a consultant in the field of sensor systems.

Dr. Meijer is a member of the Netherlands Society for Radio and Electronics. 